\title{
Surface oxygen exchange between yttria-stabilised zirconia and a low-temperature oxygen rf-plasma
}

\author{
Marcus Rohnke ${ }^{\mathrm{a}}$, Jürgen Janek ${ }^{\mathrm{a}, *}$, John A. Kilner ${ }^{\mathrm{b}}$, Richard J. Chater ${ }^{\mathrm{b}}$ \\ ${ }^{a}$ Physikalisch-Chemisches Institut, Justus-Liebig-Universität Giessen, Heinrich-Buff-Ring 58, 35392 Giessen, Germany \\ ${ }^{\mathrm{b}}$ Department of Materials, Imperial College of Science, Technology and Medicine, Prince Consort Road, London SW7 2BP, UK
}

\begin{abstract}
Isotope Exchange/Depth Profiling (IEDP) using Secondary Ion Mass Spectrometry (SIMS) has been used to determine the oxygen tracer diffusion and surface exchange coefficients of (100) oriented $9.5 \mathrm{~mol} \%$ yttria stabilised zirconia single crystals. Exchange experiments performed with molecular oxygen are compared with the exchange using an oxygen plasma. The surface exchange coefficient for specimens in a plasma is up to 100 times higher compared to measurements with normal molecular oxygen. For the exchange experiments we used an inductively coupled radio frequency (rf) oxygen plasma with a maximum radio frequency power of $250 \mathrm{~W}$. Double probe measurements and optical emission spectrometry are used for the characterisation of the plasma. The measured electron temperatures are within the range of $5-12 \mathrm{eV}$.
\end{abstract}

PACS: $52.25 ; 52.40 . \mathrm{H} ; 82.65 . \mathrm{Yh}$

Keywords: Low temperature plasma; Oxygen surface exchange; SIMS; Yttria Stabilised Zirconia (YSZ); Surface kinetics

\section{Introduction}

A knowledge of the kinetics of oxygen incorporation into (and release from) a solid electrolyte is very important for the further development of solid oxide fuel cells, membrane reactors, sensors and electrochemical promotion in heterogeneous catalysis. In particular at low temperatures the kinetics is usually relatively slow due to the participation of steps with high thermal activation. Different kinds of catalysing electrodes, for example oxide perovskites or platinum, are often used to accelerate such processes [1]. Various methods have been applied to obtain information on the kinetics and the rate-limiting step. Usually kinetic studies are performed by stoichiometry relaxation experiments and are evaluated via conductivity relaxation [2,3]. The second and often applied method is impedance spectroscopy, which offers valuable information on boundary resistances and capacities [4]. Furthermore ${ }^{18} \mathrm{O} /{ }^{16} \mathrm{O}$ isotope

\footnotetext{
* Corresponding author. Tel.: +49-641-99-34500; fax: +49-641-9934509 .

E-mail address: Juergen.Janek@phys.chemie.uni-giessen.de (J. Janek).
}

exchange experiments are also used for the quantification of the exchange kinetics [5,6]. More recently Merkle et al. investigated the oxygen incorporation kinetics of Fe-doped $\mathrm{SrTiO}_{3}$ in stoichiometry relaxation experiments using UVVis spectroscopy. They showed that the incorporation of oxygen in this case is influenced by UV irradiation with photons, which excite electron-hole pairs [7]. As Maier points out in [8], these different methods offer different information on the microscopic processes. In contrast to stoichiometry relaxation (non-equilibrium experiment) one cannot distinguish between the in- and excorporation of oxygen in the isotope exchange experiment (equilibrium experiment).

Examining the exchange mechanism of oxygen in oxides more closely we have to distinguish generally between electron-rich and electron-poor materials. In electron-rich materials with either a small band gap or sufficient ndoping, the reduction of oxygen should be relatively fast or at least not be limited by electron transfer. The reduction step might be rate limiting in electron-poor materials as is the case for all zirconia-based solid electrolytes. The incorporation of oxygen can be divided generally into several steps: Firstly, an oxygen molecule has to adsorb on the 
surface. This process is controlled mainly by the oxygen gas pressure and the oxygen sticking coefficient, and we can formulate an adsorption isotherm. The reduction of $\mathrm{O}_{2}$ molecules may proceed via different reaction channels. The following sequence for the formation of reduced species has been proposed by different authors in the literature $[9,10]$ :

$\mathrm{O}_{2, \text { ads }} \rightarrow \mathrm{O}_{2, \text { ads }}^{-} \rightarrow \mathrm{O}_{2, \text { ads }}^{2-} \rightarrow 2 \mathrm{O}_{\text {ads }}^{-} \rightarrow 2 \mathrm{O}_{\text {lattice }}^{-}$

The dissociation of the neutral and free oxygen molecule requires a high dissociation energy of $498 \mathrm{~kJ} / \mathrm{mol}$ $(5.2 \mathrm{eV})$, thus is a highly improbable step. The formation of singly and doubly charged molecular anions (superoxide and peroxide ion) reduces the dissociation energy and increases the rate of dissociation. It was shown by EPR spectroscopy that the formation of $\mathrm{O}_{2}^{-}$species takes place at room temperature [11]. Whether Eq. (1) describes the real sequence of microscopic steps at operational temperatures is yet not known. Currently there are no in situ experiments available which might prove the complete sequence.

Most surface analytical methods are restricted to UHV conditions and thus do probably not provide data relevant for oxygen exchange kinetics under atmospheric conditions. However valuable information on microscopic electrode processes in the $\mathrm{Pt} / \mathrm{YSZ}$ system has recently been obtained by photoelectron microscopy (PEEM) and microspectroscopy (SPEM) under in situ conditions [12].

Manning et al. [13] showed for yttria stabilised zirconia (YSZ), an electron-poor material, that the rate-determining step of the oxygen exchange appears to be the dissociation of oxygen above $700{ }^{\circ} \mathrm{C}$ [14]. He analysed ${ }^{18} \mathrm{O} /{ }^{16} \mathrm{O}$ exchange experiments via two different techniques: He analysed the concentration profile of ${ }^{18} \mathrm{O}$ in YSZ and also determined the composition of the gas phase during the exchange experiment. Below this temperature there must be also other slow kinetic steps, as in the gas phase analysis he detected a large amount of ${ }^{16} \mathrm{O}^{18} \mathrm{O}$ species. This mixed species is expected if the dissociation rate of oxygen molecules is fast compared to the oxygen incorporation step. Nevertheless a higher concentration of activated oxygen species on the surface should result in a higher rate of incorporation.

Based on Manning's findings, one may ask whether the direct supply with atomic oxygen increases the exchange rate. As is well known from plasma physics, oxygen plasmas often contain a significant fraction of atomic oxygen [15]. Consequently we are interested whether there is a difference in the exchange kinetics using a low temperature oxygen plasma instead of molecular oxygen.

Plasma enhanced exchange reactions are also interesting from another point of view. Recently De Ridder published careful experimental studies of the influence of surface segregation effects on the exchange kinetics of YSZ and of surface cleaning procedures (mainly polycrystaline specimens) [16]. He measured a pronounced difference in the reactivity of clean YSZ surfaces and surfaces with segregated impurities. In addition he found, that surface cleaning in an oxygen plasma combined with annealing is one of the two most efficient methods to obtain clean YSZ surfaces. As the better method De Ridder proposes the use of an atomic oxygen source.

To investigate the influence of excited oxygen species on the surface kinetics we performed isotopic exchange experiments on $9.5 \mathrm{~mol} \%$ yttria stabilised zirconia single crystals in oxygen atmospheres under thermal and plasma conditions. YSZ is an excellent oxygen ion conductor at high temperatures and has a very low electronic conductivity. For simplification the transference number of the ions can be set equal to 1 [17]. The ionic mobility depends strongly on the temperature due to its thermally activated character and in most applications YSZ can only be used above $350{ }^{\circ} \mathrm{C}$. The material has a band gap in the order of $5.2 \mathrm{eV}$ [18], thus only UV radiation with a wave length of less than $240 \mathrm{~nm}$ may influence the kinetics by electron-hole formation.

\section{Experimental}

\subsection{Experimental setup}

The experiments were performed in a self-constructed rf-plasma reactor (see Fig. 1). The reactor consists of a $\mathrm{SiO}_{2}$ glass tube, which is fixed between two stainless steel plates. An oil rotation pump generates a vacuum down to $0.1 \mathrm{~Pa}$. The reaction gas is supplied by mass flow controllers (MKS, Munich) which allow to set gas flows down to $0.3 \mathrm{ml} / \mathrm{min}$ with different gas mixtures. The pressure is measured with a baratron manometer and the MKS control unit $647 \mathrm{~B}$ held the pressure constant within $\pm 0.1 \%$. It is possible to run the reactor either as batch reactor or as continuous stirred tank reactor. The radio frequency is produced by a radio frequency generator PFG 300 (Hüttinger, Freiburg) and is coupled to the gas phase inductively via a copper coil, which is placed around the glass tube. The frequency is $13.56 \mathrm{MHz}$ and the maximum rf-power $300 \mathrm{~W}$. A matching network PFM 1500 A (Hüttinger, Freiburg) is used to optimise the coupling, i.e. to reduce the power loss. In the bottom plate of the reactor is a flange to which different sample holders can be attached. For the tracer experiments we used a self-made heating stage with an integrated shielded thermocouple. The upper plate has a flange for a double probe. Alternatively a glass window can be fixed to the upper flange, which allows measurement of the sample surface temperature using an infrared sensor. The temperature can be varied continuously from room temperature to $800{ }^{\circ} \mathrm{C}$. In Fig. 2 a schematic drawing of the rf- 


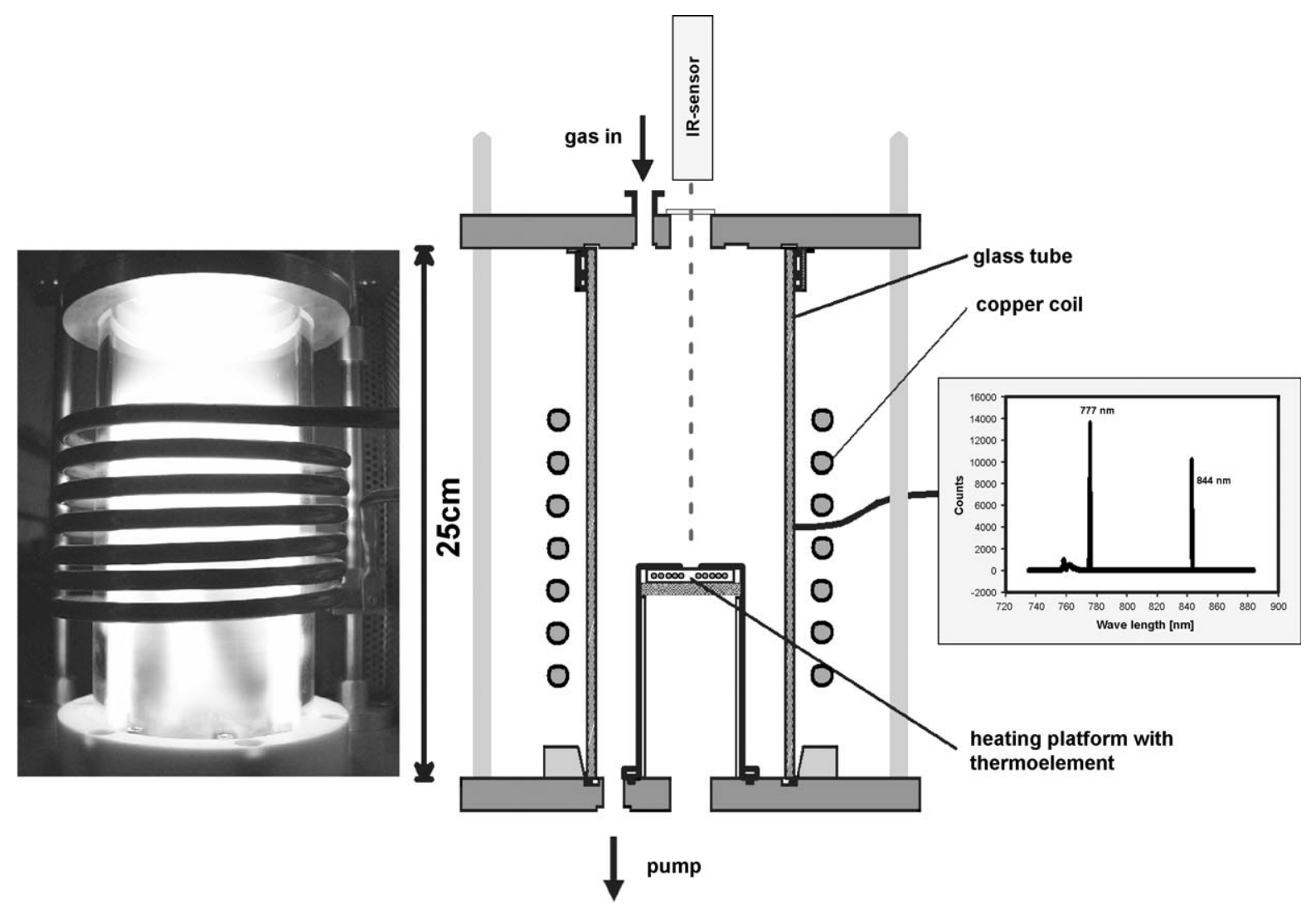

Fig. 1. Sketch of the self constructed rf-plasma reactor. The photograph shows a burning oxygen plasma at $300 \mathrm{~W}$ and $10 \mathrm{~Pa}$ oxygen pressure.

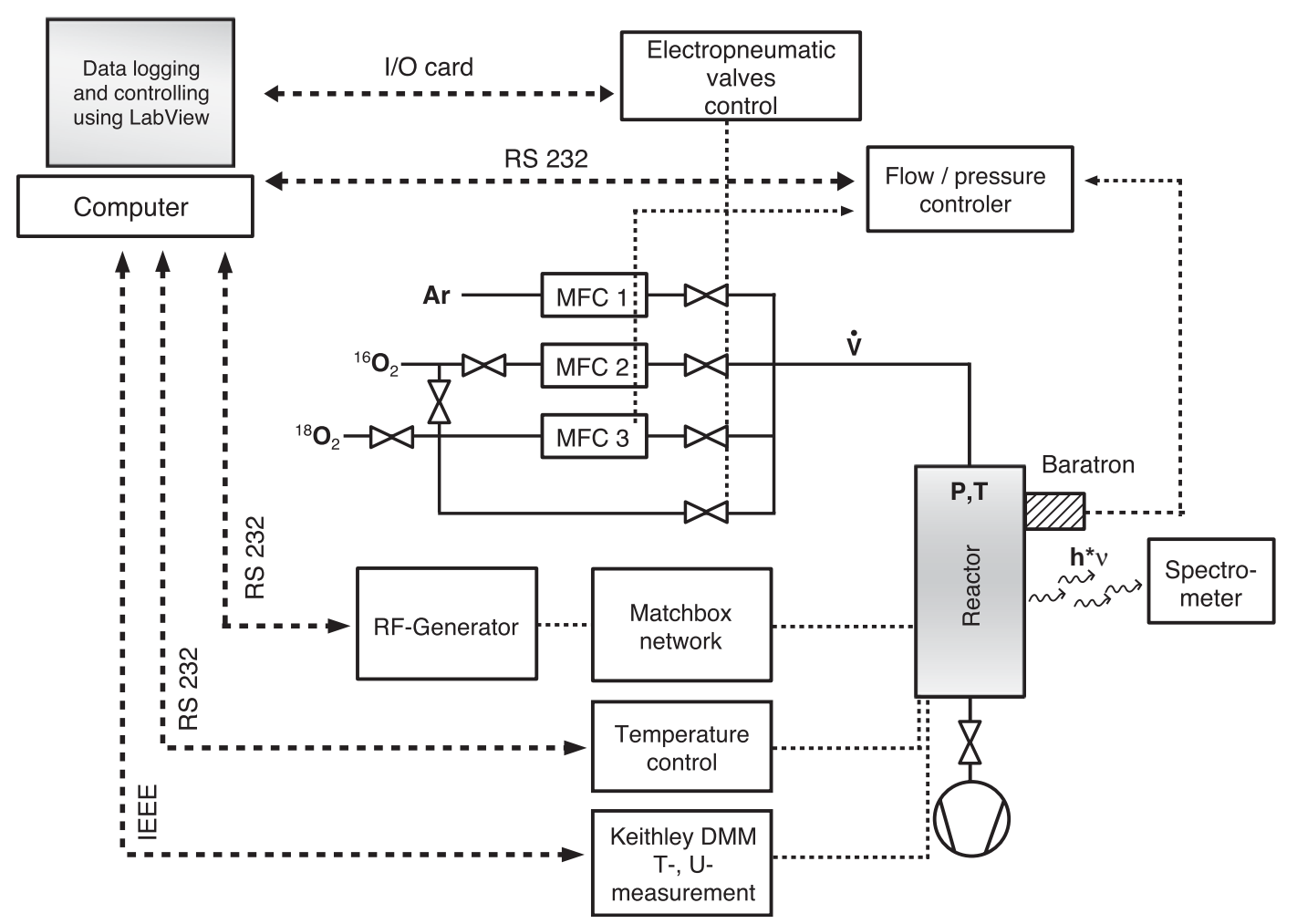

Fig. 2. Technical mapping of the rf-equipment. 
equipment is shown. The system is completely computercontrolled employing LabView ${ }^{\circledR}$ (National Instruments) as a graphical programming language.

\subsection{Plasma diagnostics}

Since the plasma parameters depend strongly on the geometry of the reactor it was necessary to characterise the oxygen plasma before the exchange experiments. Using a shielded thermocouple $(0.5 \mathrm{~mm}$ diameter $)$ we measured the kinetic temperature of (neutral and ionic) particles in the oxygen plasma in $z$-direction (parallel to the axis of the glass tube) as a function of the rf-power. Furthermore the optical and UV-plasma emission was determined by optical emission spectrometry (MS257, LOT-Oriel, Darmstadt). The emission was transferred via fibre optics from the glass tube to the spectrometer. The analysis offers qualitative information on changes in the atomic oxygen concentration. It was not possible to measure the atomic oxygen concentration quantitatively in absolute numbers.

In addition we realised double probe measurements with a self made double probe. The double probe consists of two Pt-wires (0.3 mm diameter, $10 \mathrm{~mm}$ long, $10 \mathrm{~mm}$ distance). For the $I-U$ measurements we used a sourcemeter (Keithley, model 2400).

\subsection{Sample preparation}

In order to obtain reliable data from the SIMS measurements, the sample surface had to be as smooth as possible. The 100-orientated YSZ (9.5 mol\%, TBLKelpin, Neuhausen) single crystals were polished with different grades of diamond abrasive down to $0.25 \mu \mathrm{m}$. The final polishing was done with $0.02 \mu \mathrm{m} \mathrm{SiO}_{2}$ abrasive. Prior to oxygen exchange all samples were ultrasonic-cleaned in acetone and afterwards plasma-cleaned for $600 \mathrm{~s}$ (150 W rf-power at $10 \mathrm{~Pa}$ oxygen pressure), cf. [19] for a comparison of different cleaning procedures. For the SIMS analysis cross sections of the samples were prepared after each exchange experiment by embedding, cutting with a low speed saw and polishing as already described.

\subsection{Isotope exchange and SIMS}

The plasma reactor was evacuated to a pressure lower than $0.5 \mathrm{~Pa}$ before normal oxygen $(99.8 \%$ purity) was introduced. The samples were then annealed under a continuous flow of oxygen at the required temperature for two days and thereafter plasma-cleaned. The plasma apparatus was then evacuated to the base pressure and subsequently the isotope ${ }^{18} \mathrm{O}(99.9 \%$, Linde) was let in. After the isotopic annealing the samples were cooled down rapidly and the oxygen atmosphere was exchanged by a high argon flux.
For SIMS analysis we used an Atomika 6500 ion microprobe, equipped with a quadrupole mass analyser. Primary $\mathrm{Ar}^{+}$ions with an energy of $8 \mathrm{keV}$ were used. To prevent sample charging during the analysis, the samples were co-bombarded with a $1 \mathrm{keV}$ electron beam. More details of the isotopic exchange SIMS technique are reported by Chater et al. [5].

\section{Results}

\subsection{Temperature profile}

Compared to thermal experiments the thermodynamic characterisation of a plasma experiment suffers from a major disadvantage. Any low temperature plasma is a non-equilibrium system, which means that the electron temperature differs significantly from the temperature of the ions and the neutral particles. Normally the temperature of the electrons is up to 100 times higher than the temperatures of ions and neutrals [20]. Of main interest for plasma-solid reactions is the thermodynamic temperature of a sample immersed into the plasma.

The temperature profile measured with a shielded thermocouple is depicted in Fig. 3. The temperature increases with increasing rf-power up to temperatures of $650{ }^{\circ} \mathrm{C}$. The temperature is also varying with the $z$-position. The hottest areas are at the ends of the copper coil. In the middle of the coil the temperature increase is relatively low. At the position of the heating stage it is definitely lower than 500 ${ }^{\circ} \mathrm{C}$. This is very important for the exchange experiments, which require temperatures between 500 and $600{ }^{\circ} \mathrm{C}$. Thus only minor external heating is required in order to maintain the defined experimental conditions.

\subsection{Double probe measurements}

For generating the plasma a $13.56 \mathrm{MHz}$ signal is used and the radio frequency power is coupled to the gas inductively via a copper coil. Due to their high masses all oxygen particles are virtually unaffected by the alternating field and maintain a Maxwell-Boltzmann-type energy distribution. In contrast the electrons are accelerated to much higher velocities, which correspond to high kinetic electron temperatures up to $12 \mathrm{eV}$. The energy of neutral particles is influenced slightly by the impact of electrons, thus they are in thermal equilibrium with the walls of the reactor. Typical temperatures of neutrals are in the order of $0.05 \mathrm{eV}$ (room temperature) and the ion temperature is usually higher (approximately $0.5 \mathrm{eV}$ ). Low temperature oxygen plasmas are weakly ionised. The degree of ionisation $\alpha$ is smaller than $1 \cdot 10^{-5}$ and the plasma density $n$ corresponds to $5 \cdot 10^{11} / \mathrm{cm}^{3}$ at $10 \mathrm{~Pa}$. The electron temperature $T_{\mathrm{e}}$, the plasma potential $V_{\mathrm{P}}$, the ion density $I_{\mathrm{s}}$ and the degree of ionisation $\alpha$ can be evaluated from double probe measurements [21]. 


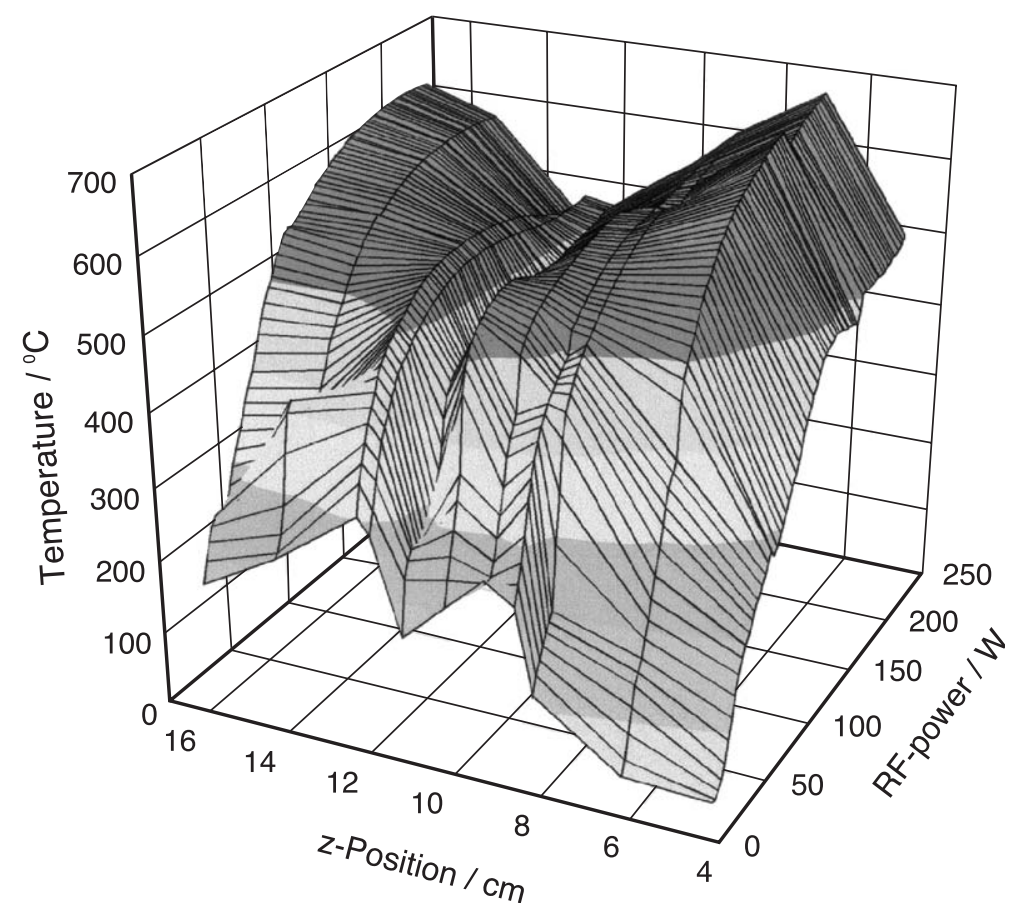

Fig. 3. Temperature profile of the oxygen plasma at different rf-powers and $z$-positions. Oxygen pressure of $p\left(\mathrm{O}_{2}\right)=200 \mathrm{~Pa}$.

The voltage-current characteristics of the double probe are analysed with the following expression, which was firstly derived by Johnson and Malter [22,23]:

$$
\begin{aligned}
\frac{k_{\mathrm{B}} T_{\mathrm{e}}}{e_{0}}= & {\left[2\left(\frac{\mathrm{d} I_{\mathrm{d}}}{\mathrm{d} U_{\mathrm{d}}}\right)_{\mathrm{fl}}-\frac{1}{2}\left(\frac{\mathrm{d} I_{\mathrm{pi} 1}}{\mathrm{~d} U}+\frac{\mathrm{d} I_{\mathrm{pi} 2}}{\mathrm{~d} U}\right)_{\mathrm{fl}}\right]^{-1} } \\
& \cdot \frac{2\left(I_{\mathrm{pi} 1}\right)_{\mathrm{fl}}\left(I_{\mathrm{pi} 2}\right)_{\mathrm{fl}}}{\left(I_{\mathrm{p} 1}\right)_{\mathrm{fl}}+\left(I_{\mathrm{pi} 2}\right)_{\mathrm{fl}}}
\end{aligned}
$$

In this equation $k_{\mathrm{B}}$ is the Boltzmann constant, $T_{\mathrm{e}}$ the electron temperature, $e_{0}$ the elementary charge, the indices 1 and 2 denote probes 1 and 2, the subscript fl denotes the floating potential, and $\left(I_{\mathrm{pi} 1,2}\right)_{\mathrm{fl}}$ denotes the extrapolated value of the ion current at $U_{\mathrm{d}}=0 \mathrm{~V}$. From Eq. (2) we obtain the electron temperature $T_{\mathrm{e}}$ by a fit to the experimental data. An analysis of the voltage-current plot leads to comparable results using the theory of Chen [24]. If the probe surface areas of both probes are equal, the ion density $n_{\mathrm{i}}$ is given as [25]:

$n_{\mathrm{i}}=\frac{I_{\mathrm{s}}}{0.4 \cdot e_{0} \cdot A} \cdot \sqrt{\frac{m_{\mathrm{i}}}{2 \cdot e_{0} U}}$

Here $I_{\mathrm{s}}$ denotes the ion saturation current, $A$ is the electrode area, $m_{\mathrm{i}}$ is the mass of the ions and $e_{0} U$ is the electron temperature in $\mathrm{eV}$. Most ions in the oxygen plasma are singly charged, and the electron density is thus equal to the ion density. The degree of ionisation $\alpha$ is the ratio of the ion density and the total particle density in the reactor, which can be calculated from the pressure. In Fig. 4 the electron temperature is plotted versus the radio frequency power. The electron temperature increases nearly linear with the power. It has a value in the range $5 \mathrm{eV}<T_{\mathrm{e}}<10 \mathrm{eV}$. Both analysis techniques (Johnson, Malter and Chen) lead to comparable results. The dependence of the degree of ionisation on the rf-power is plotted in Fig. 5. $\alpha$ is in the order of $10^{-6}$ and is also increasing linearly with increasing power. Another important aspect is the variation of the electron temperature with the $z$-position in the reactor, which is depicted in Fig. 6. The electron temperature shows a maximum in the middle of the copper coil, which is approximately at $z=12 \mathrm{~cm}$ in a region with an electron energy of $T_{\mathrm{e}} \cong 10 \mathrm{eV}$. The sample position is chosen as $z=10.2 \mathrm{~cm}$. For the double probe measurements we had to remove the sample holder. Thus the electron temperature at the sample in the exchange experiment is probably not the same compared with the results of the double probe measurements. However these measurements are useful to get information on the magnitude of $T_{\mathrm{e}}$. The plasma potential $V_{\mathrm{P}}$ extracted from the voltage-current curve is in the range $20-25 \mathrm{~V}$ but also depends on the rf-power and the gas pressure.

\subsection{Optical emission spectroscopy}

In addition to particles with a very short lifetime the following species can be found in an inductively coupled low temperature radio frequency oxygen plasma [15]: $\mathrm{O}$, 


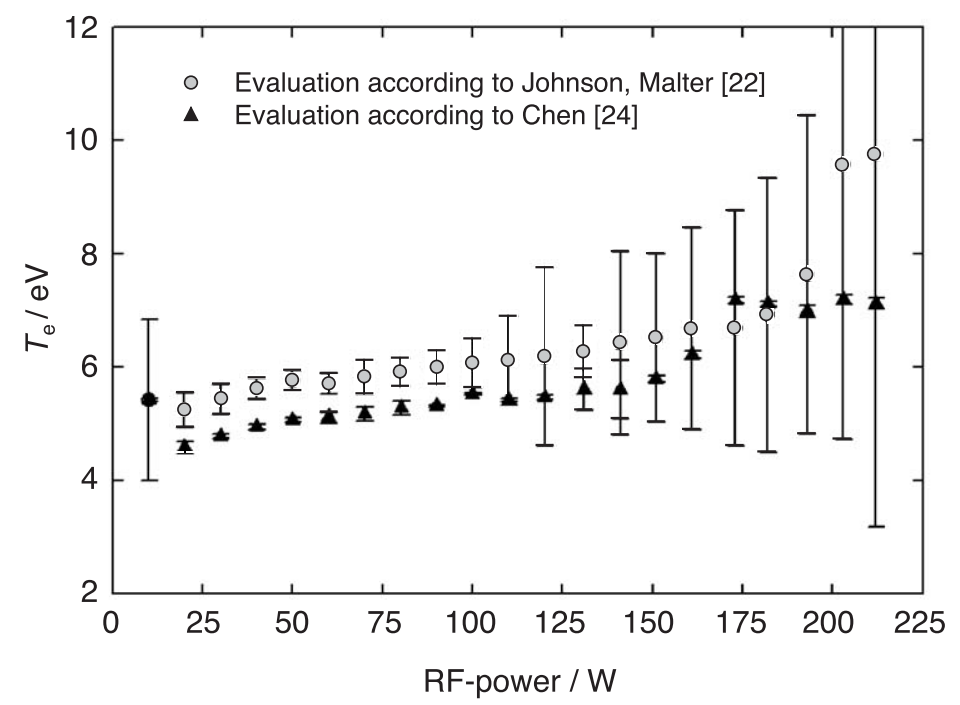

Fig. 4. The electron temperature evaluated from the double probe measurements according to Johnson, Malter and Chen are plotted versus the rf-power. $p\left(\mathrm{O}_{2}\right)=200 \mathrm{~Pa}, z=6.4 \mathrm{~cm}$.

$\mathrm{O}_{2}, \mathrm{O}_{3}, \mathrm{O}^{+}, \mathrm{O}_{2}^{+}, \mathrm{O}^{-}, \mathrm{O}_{2}^{-}, \mathrm{O}_{3}^{-}, \mathrm{e}^{-}$. As shown by Lee et al. [26] the dominant species at $10 \mathrm{~Pa}$ is indeed atomic oxygen. The degree of ionisation and the concentration of ionised species are relatively low at this pressure.

Oxygen plasmas are glowing shallow blue. Normally this plasma-induced emission shows two intensive emission lines at 777 and $844 \mathrm{~nm}$. These lines are assigned to the $3 p^{5} P-3 s^{5} S$ transition at $777.4 \mathrm{~nm}$ and the $3 p^{3} P-3 s^{3} S$ transition at $844.6 \mathrm{~nm}$ of excited atomic oxygen. It was shown by Laser Induced Fluorescence (LIF) experiments that two different excitation processes can produce the emitting oxygen atom [27]: electron impact excitation and dissociative excitation. The excitation mechanism depends on the plasma conditions and thus varies with the experimental setup.

$\mathrm{e}^{-}+\mathrm{O} \rightarrow \mathrm{O}^{*}+\mathrm{e}^{-}$
$\mathrm{e}^{-}+\mathrm{O}_{2} \rightarrow \mathrm{O}^{*}+\mathrm{O}+\mathrm{e}^{-}$

The emission is produced by the process

O* $\stackrel{777 \mathrm{~nm}, 844 \mathrm{~nm}}{\longrightarrow} \mathrm{O}$

Due to the emission it is very easy to monitor the excited atomic oxygen concentration in the reactor qualitatively using an optical emission spectrometer. In a first order approximation the emission intensity is proportional

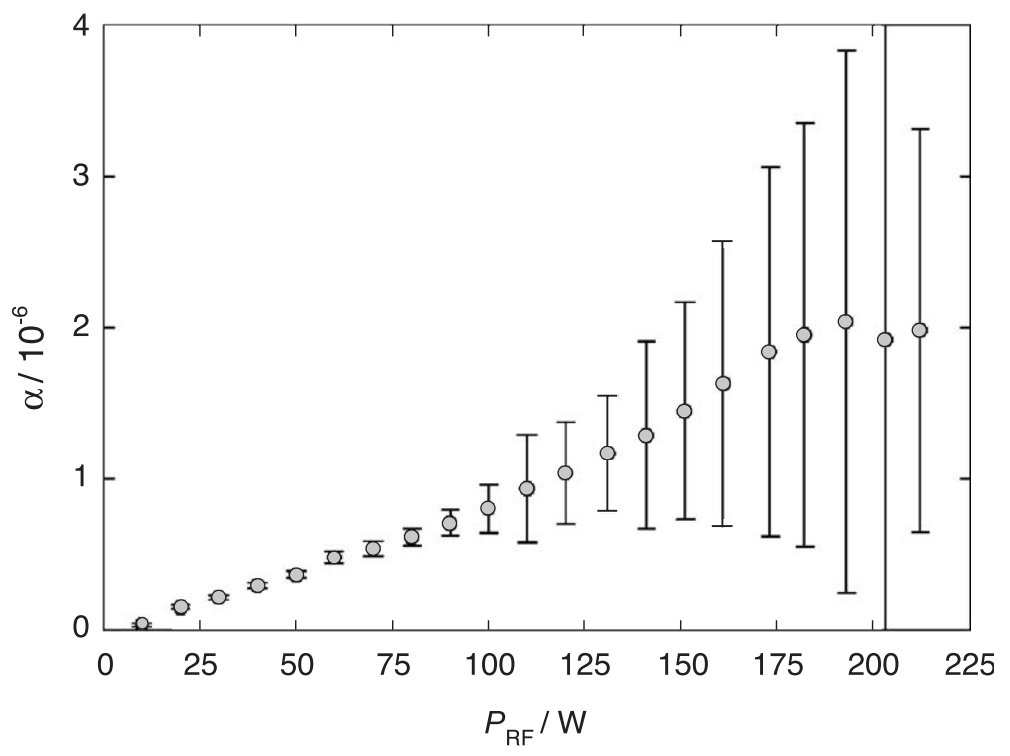

Fig. 5. Degree of ionisation $\alpha$ plotted versus the rf-power. $p\left(\mathrm{O}_{2}\right)=200 \mathrm{~Pa}, z=6.4 \mathrm{~cm}$. 


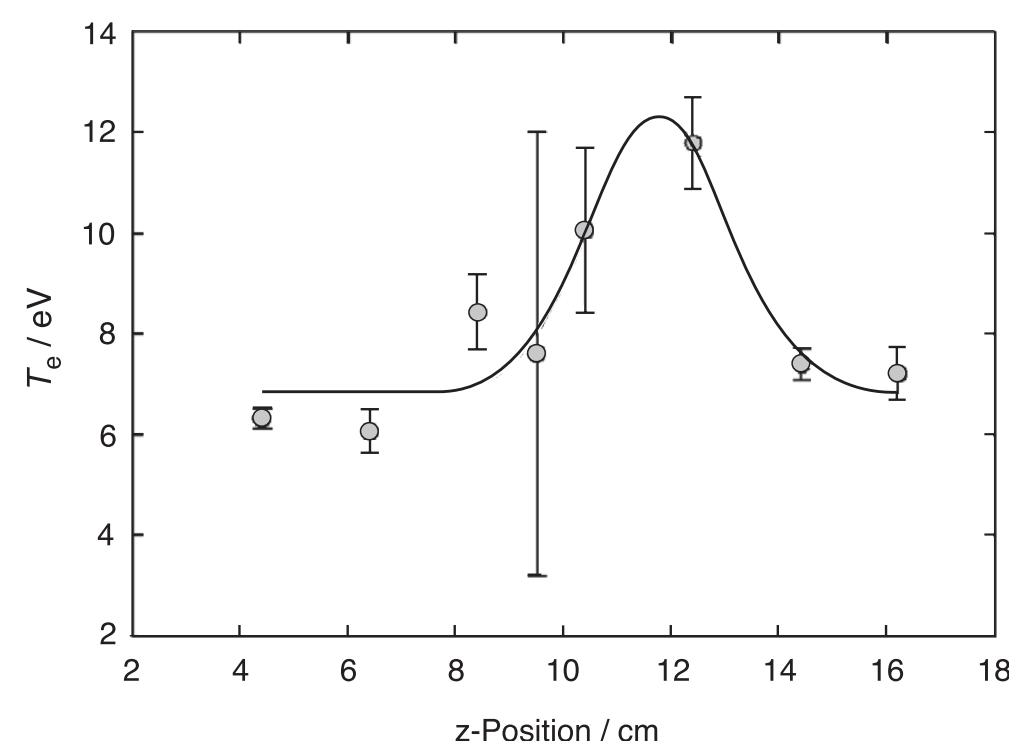

Fig. 6. Variation of the electron temperature at different $z$-positions in an oxygen plasma.

to the concentration of atomic oxygen. For the determination of an absolute concentration of excited oxygen it is necessary to calibrate the spectrometer, i.e. a reactor containing a known concentration of atomic oxygen is required. Alternatively the actinometric technique can be used. For this purpose a small amount of an actinometer gas is introduced into the plasma with the process gas. The emission from an excited state of the actinometer gas into the ground state is measured. For $\mathrm{O}_{2}$ plasmas this actinometer gas is usually Ar [28]. The emission intensities of $\mathrm{Ar}^{*}$ and $\mathrm{O}^{*}$ are measured simultaneously. Normally the intensity of the emission is proportional to the concentration of the observed species. The ratio of both emission intensities, the actinometer $\mathrm{Ar}$ and the process gas $\mathrm{O}_{2}$, gives a measure of the atomic oxygen density. This technique requires four conditions, which have to be fulfilled: (1) The thresholds of the actinometrical and the observed gas must be nearly the same. (2) Both gases show the same excitation process. (3) Excitation cross-sections for both gases have similar energy dependence. (4) Equal fractions of the excited species decay radiatively.

In our case the UV-Vis spectra of the plasma induced emission shows relatively weak emission lines of $\mathrm{O}^{+}, \mathrm{O}_{2}^{+}$ and the forbidden $\mathrm{O}_{2}$ rotation transition at $760 \mathrm{~nm}$. Besides the two intensive lines generated by excited atomic oxygen at $777 \mathrm{~nm}$ and $844 \mathrm{~nm}$ are measured. The peak-area of these two peaks is plotted in Fig. 7

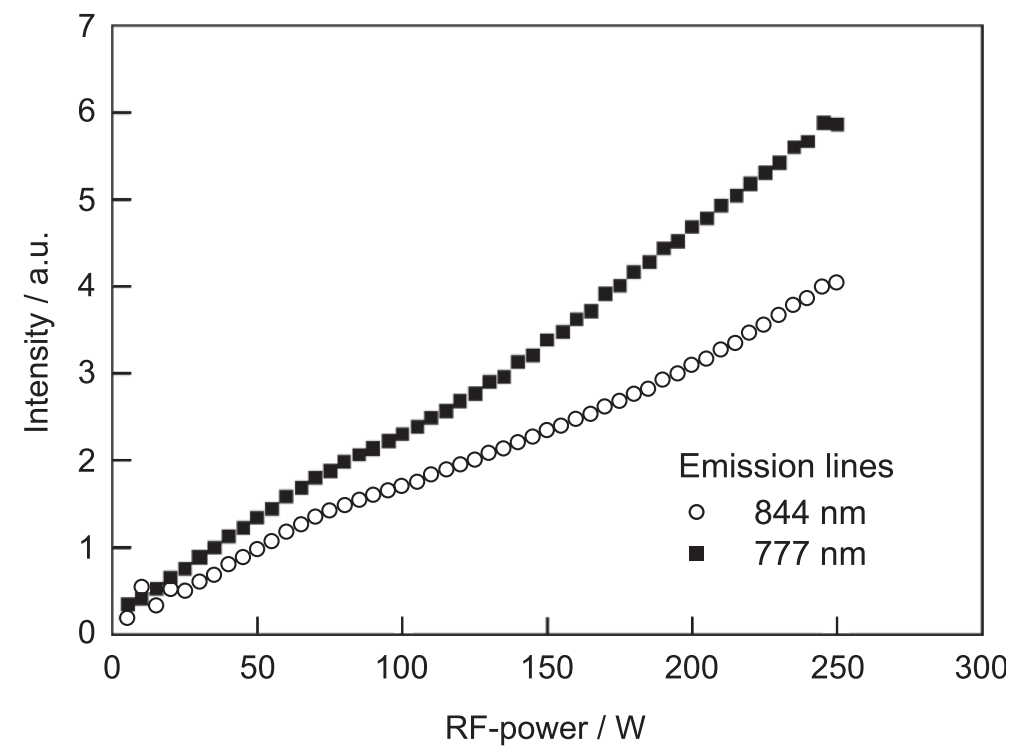

Fig. 7. Intensity of the $777 \mathrm{~nm}$ and the $844 \mathrm{~nm}$ peak of the UV-Vis spectra as a function of the rf-power. 


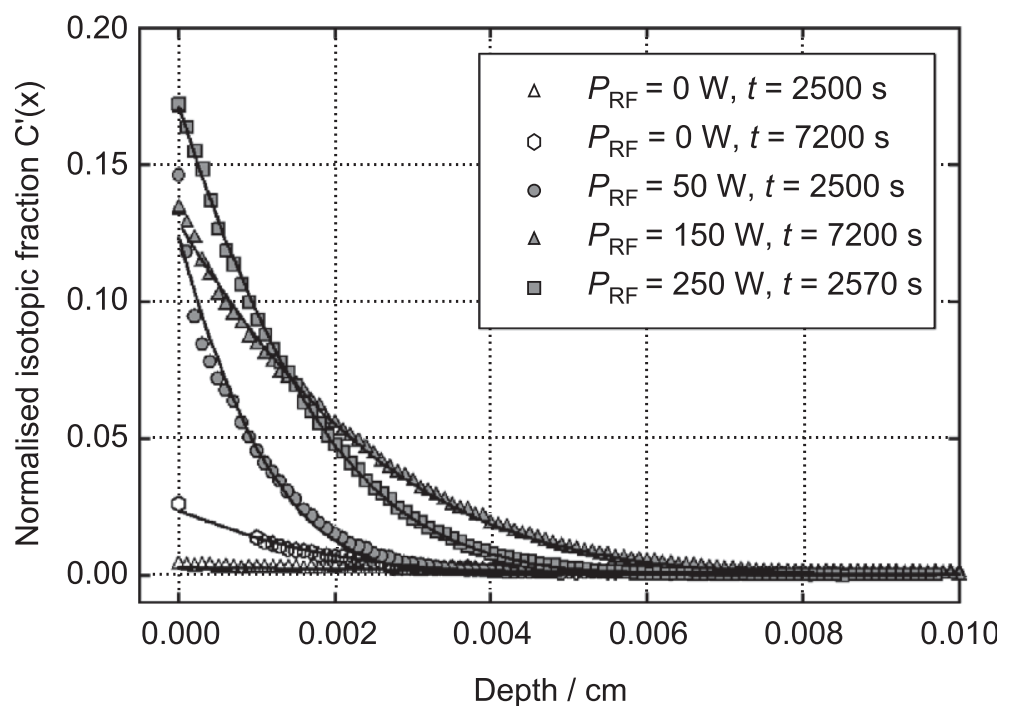

Fig. 8. Experimental raw data from the SIMS measurements. The normalised isotopic ratio is plotted versus the sample depth. The solid lines are the simulated diffusion profiles. $T=600{ }^{\circ} \mathrm{C}$.

versus the radio frequency power. The change of the concentration of excited atomic oxygen, which is proportional to the concentration of atomic oxygen, with increasing rf-power is observed. The results are identical for both emission lines.

In order to obtain the absolute oxygen concentration we tried to use the actinometric technique. Argon was chosen as actinometer gas. As observed in these experiments the oxygen peaks at $777 \mathrm{~nm}$ and at $844 \mathrm{~nm}$ are produced by both possible excitation processes (electron impact and dissociation excitation). Therefore it is not possible to apply the actinometric technique. The condition number two failed, and we obtain only relative information from emission spectrometry.

\subsection{SIMS analysis of diffusion profiles}

Fig. 8 shows diffusion profiles of IEDP experiments with and without plasma treatment. In this graph the isotopic ratio is plotted versus the sample depth. The zero position is assigned to the plasma-exposed surface on the YSZ specimen. The experiments in Fig. 8 were performed at $600{ }^{\circ} \mathrm{C}$. It can be seen clearly that there is built in significantly more oxygen from the plasma despite of even shorter annealing times. The area underneath the line, which corresponds to the amount of oxygen, is definitely larger.

IEDP experiments were made at 500, 550 and $600{ }^{\circ} \mathrm{C}$ at different radio frequency powers. Exchange experiments below $500{ }^{\circ} \mathrm{C}$ were not performed, since otherwise cooling

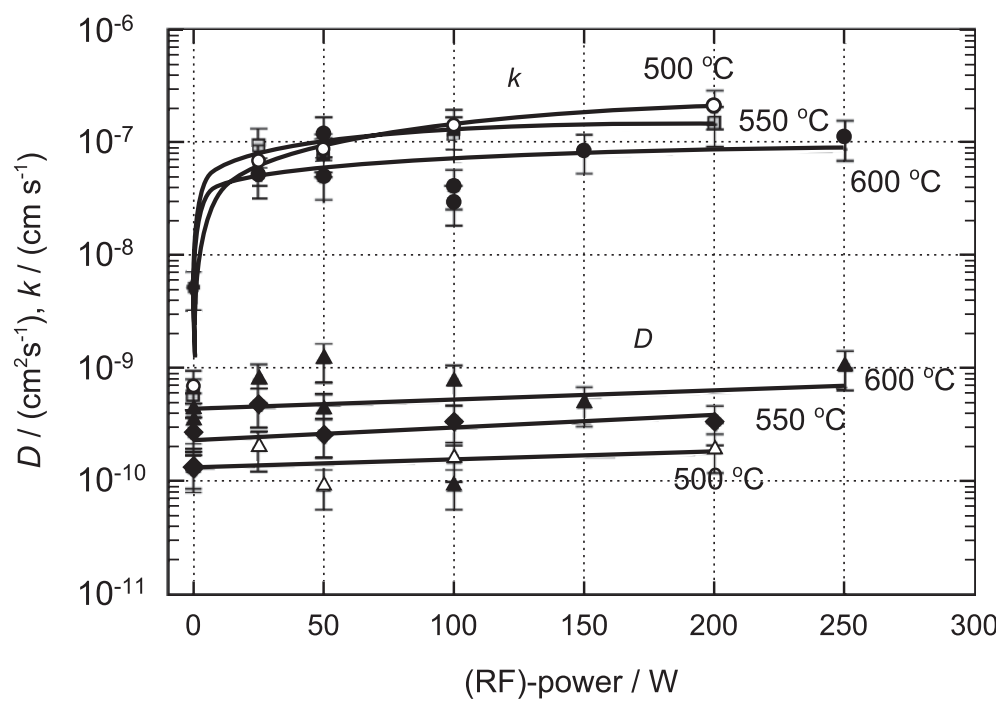

Fig. 9. Diffusion coefficients $D$ and rate coefficients $k$ got from IEDP measurements. The solid lines are produced by the spline function of Sigama Plot. They are only for visualisation. 


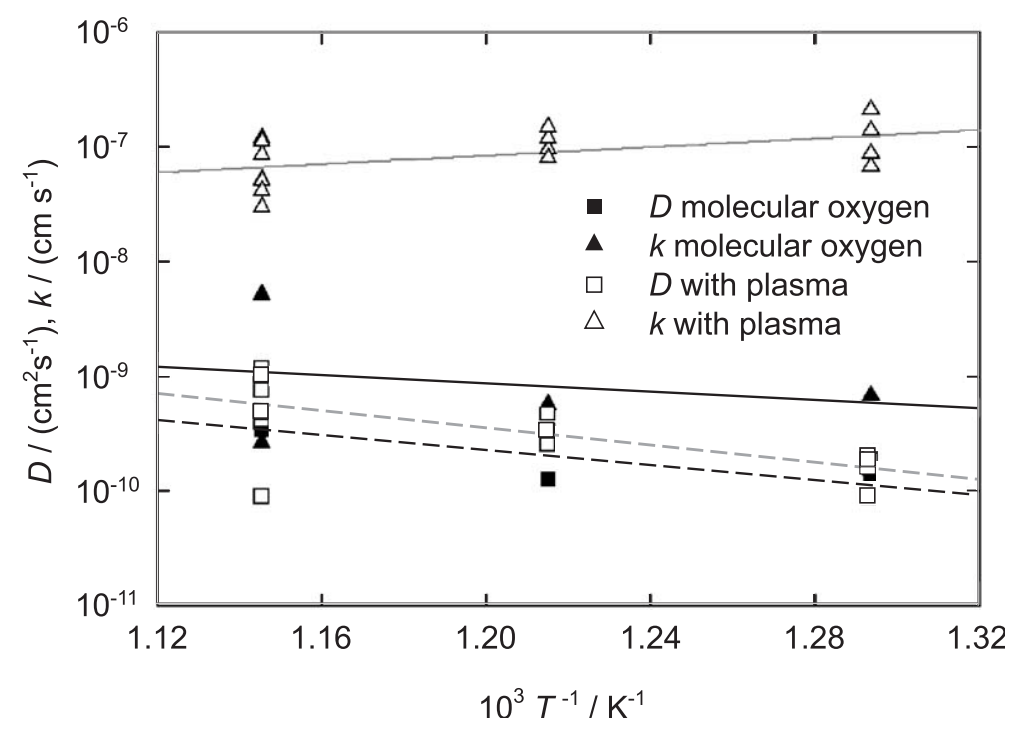

Fig. 10. Arrehnius plot of the diffusion $D$ and rate coefficients $k$. Dotted line - regression lines diffusion coefficients. Solid lines - regression lines exchange coefficients.

of the samples would have been necessary. Temperatures above $600{ }^{\circ} \mathrm{C}$ were not chosen in order to reduce temperature gradients between the sample surface and the thermocouple.

In Fig. 9 the diffusion coefficients and the rate constants for the exchange experiments at different plasma powers for 500, 550 and $600{ }^{\circ} \mathrm{C}$ are depicted. These data were evaluated by the use of Eq. (9) from the diffusion profiles. The diffusion coefficients are constant within an error of $\pm 32 \%$. A linear regression shows nevertheless that the diffusion coefficients become slightly higher with increasing rf-power. This is due to the increasing heating power of the plasma. The rate constants show a completely different behaviour. The rate constants of the plasma experiments are up to 100 times higher than those of thermal experi- ments. The effect of the plasma becomes smaller with increasing temperature. At $600{ }^{\circ} \mathrm{C}$ the ratio of the rate constants with and without plasma is only a factor 10 . If we would try to generate the same effect via heating, we had to raise the temperature by a few hundred Kelvin. The influence of the increasing plasma power on the rate constants is strongly non-linear. It appears that already a minor plasma power is sufficient to obtain a maximum effect.

In Fig. 10 an Arrhenius plot of the data from Fig. 9 is depicted. For simplification the data points for different plasma powers are grouped together. The plot clearly shows the significant change of the exchange constants upon plasma treatment. Due to the small number of experimental data we did not extract activation energies from the regression lines. In Fig. 11 we plotted the obtained rate and

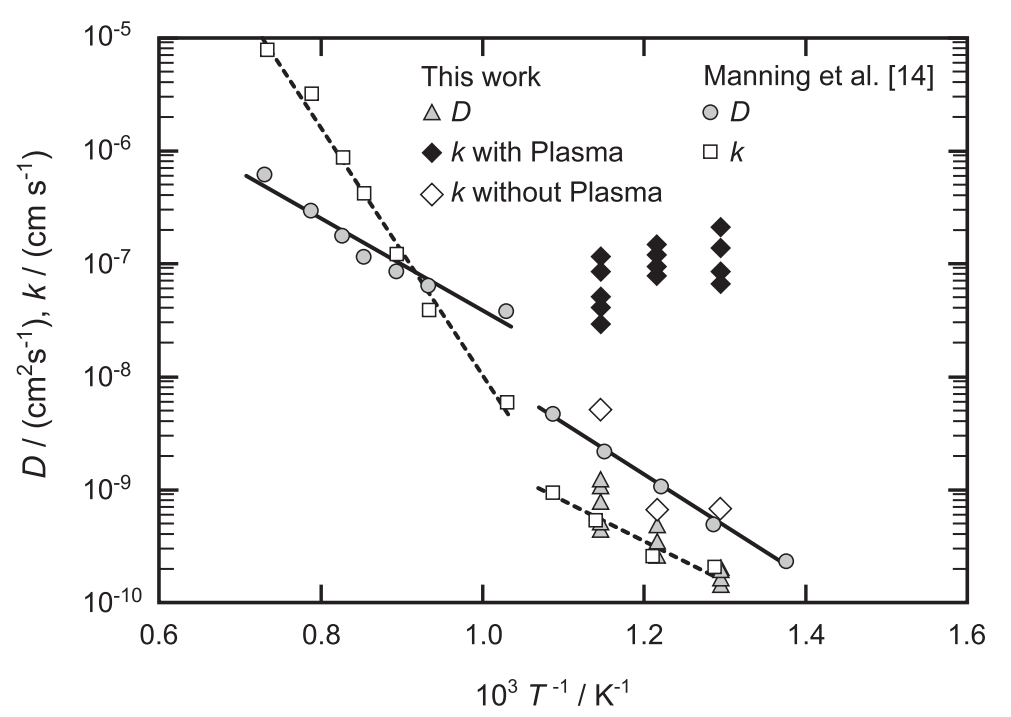

Fig. 11. Arrhenius plot of the rate constants $k$ and the diffusion coefficients $D$ obtained from own experiments in comparison with values printed in Ref. [14]. The exchange and diffusions coefficients of experiments with oxygen plasma are measured at different plasma rf-powers. 
diffusion coefficients in comparison with values received by Manning et al. from exchange experiments with molecular oxygen at single crystalline YSZ ((100)-orientation) [14]. As can be seen, our diffusion coefficients are slightly smaller than the literature values. In contrast our rate constants are definitely higher, which could be due to the plasma cleaning step before each exchange experiment. The exchange rates within the plasma are magnitudes higher.

\subsection{Data analysis of SIMS profiles}

For the analysis of the SIMS data we assume a kinetic of first order for the gas/solid and plasma/solid interface, respectively. Therefore we formulate the expression:

$k\left(C_{\mathrm{O}}-C_{\mathrm{S}}\right)=-\left.D \frac{\partial C(x)}{\partial x}\right|_{x=0}$

Here $k$ is the surface exchange coefficient (first order rate constant), $D$ is the self-diffusion coefficient, $C_{\mathrm{O}}$ is the ${ }^{18} \mathrm{O}$ concentration of adsorbed oxygen on the surface, $C_{\mathrm{S}}$ the ${ }^{18} \mathrm{O}$ concentration in the first lattice layers of the surface. The ${ }^{18} \mathrm{O}$ concentration $C_{\mathrm{O}}$ is in equilibrium with the ${ }^{18} \mathrm{O}$ concentration $C_{\mathrm{g}}$ in the gas. For simplification $C_{\mathrm{O}}$ is equated with $C_{\mathrm{g}}$. Taking Eq. (1) as boundary condition for Fick's second law of diffusion

$$
\frac{\partial C(x)}{\partial t}=D\left(\frac{\partial^{2} C(x)}{\partial x^{2}}\right)
$$

The solution of the differential Eq. (2) for diffusion into a semi-infinite medium with the initially ${ }^{18} \mathrm{O}$ concentration $C_{\mathrm{bg}}$ in the material is given by Crank [29] as

$$
\begin{aligned}
\frac{C(x)-C_{\mathrm{bg}}}{C_{\mathrm{g}}-C_{\mathrm{bg}}}= & \operatorname{erfc}\left\{\frac{x}{2 \sqrt{D t}}\right\}-\exp \left(h x+h^{2} D_{\mathrm{T}} t\right) \\
& \times \operatorname{erfc}\left\{\frac{x}{2 \sqrt{D t}}+h \sqrt{D t}\right\}
\end{aligned}
$$

with $h=k / D . C_{\mathrm{bg}}$ is the natural background level of ${ }^{18} \mathrm{O}$. In order to extract the parameters $k$ and $D$ from the profiles a non-linear least squares fit based upon Eq. (9) was used to determine $D$ and $k$.

\section{Discussion}

The main results of our experimental study can be summarised as follows:

1. A significant increase of ${ }^{18} \mathrm{O} /{ }^{16} \mathrm{O}$ exchange rate is observed upon the presence of an oxygen plasma.

2. The relation between plasma power (i.e. degree of ionisation) and rate increase appears to be strongly non-linear. Saturation is already observed at $25 \mathrm{~W}$. However, it might also be possible that even a smaller plasma power is sufficient to cause a strong effect, which is then only slightly improving with additional power. Below $25 \mathrm{~W}$ the plasma becomes unstable, and thus, the experiments had to be restricted to powers above $25 \mathrm{~W}$.

Any model for oxygen exchange from a plasma has to explain these three observations. In the following we will firstly discuss different aspects of plasma/solid interaction, which may all contribute to the enhanced surface reactivity to a varying degree.

\subsection{Plasma cleaning/surface structure}

As it is well known in surface science the atomic surface structure and the surface coverage with impurities play a crucial role for the surface reactivity. Of course oxygen exchange also depends on the number of reactive surface sites, and thus, on the microscopic nature of the oxide surface. But despite the importance of a reproducible surface preparation only a few studies on the influence of surface structure and contamination have yet been reported. Probably the most comprehensive information is available for the surface reactivity of $\mathrm{SrTiO}_{3}$. It has been shown by Denk [30] that the surfaces of freshly cleaved $\mathrm{SrTiO}_{3}$ crystals have considerably higher exchange coefficients than aged (relaxed) surfaces. Whether this effect is due to a plain structural relaxation of the surface atoms or due to a growing surface contamination (e.g. with water) with time is yet not clear.

Regardless of the results of Denk, De Ridder investigated different cleaning procedures on YSZ surfaces using low energy ion scattering (LEIS) for surface analysis on an atomic scale with respect to depth. He shows that the combination of thermal annealing and atomic oxygen treatment before an exchange experiment gives the cleanest surfaces. The maximum cleaning effect is reached after a treatment of $200 \mathrm{~s}$. Longer cleaning times do not lead to a cleaner surface [19].

The treatment of materials with plasmas is a widely used method for the activation of surfaces [25]. Thus polymer foils are often exposed shortly to plasmas in order to improve their adhesive properties. Often plasma cleaning of solid substrates is used before thin films are deposited either by sputtering or vapour deposition [31].

Indeed we observed that plasma cleaned surfaces usually have a higher surface concentration of ${ }^{18} \mathrm{O}$ after the exchange experiment, i.e. we assume that the incorporation into the lattice is faster for plasma cleaned surfaces. In our experiments we excluded this plain cleaning effect of the plasma by a similar treatment of all samples before the exchange experiments. Each sample was cleaned in an oxygen plasma before either a thermal or a plasma exchange experiment was performed. Thus the initial surface state of all samples was identical. We cannot exclude the possibility that the continuous exposure to reactive particles during the subsequent 
plasma exchange experiment prevents an ageing of the fresh surfaces, and thus, leads to an optimised surface reactivity. But since all exchange experiments took no longer than $4 \mathrm{~h}$, any surface relaxation or contamination should be very fast in order to become significant. In the current state of understanding we thus exclude a relevant contribution of continuous plasma cleaning to the improved surface kinetics. Regarding the importance of surface reactivity it might nevertheless be interesting to investigate a plasma cleaning effect more intensively.

Along with the cleaning effect we have to discuss a possible surface segregation of impurities. Hughes measured high concentrations of $\mathrm{Y}, \mathrm{Si}, \mathrm{Na}$ and $\mathrm{Fe}$ on the surfaces of YSZ single crystals, which were annealed at temperatures from 900 up to $1500{ }^{\circ} \mathrm{C}$ [32]. This study was extended by De Ridder to lower temperatures. He observed segregation of $\mathrm{Y}_{2} \mathrm{O}_{3}$ in the surface near region of polycrystalline YSZ at temperatures above $600{ }^{\circ} \mathrm{C}$ and segregation of $\mathrm{Na}, \mathrm{Si}$ and $\mathrm{Fe}$ at temperatures higher than $1000{ }^{\circ} \mathrm{C}$. In contrast to these findings the segregation in $\mathrm{YSZ}$ single crystals is much slower because of missing grain boundaries as fast diffusion paths [33]. In the present study our maximum exchange temperature is $600{ }^{\circ} \mathrm{C}$. For this reason segregation effects will play no role after the plasmacleaning step.

\subsection{UV radiation}

As already indicated the incorporation of oxygen into an oxide includes the reduction of the oxygen molecules by the supply of four electrons from the oxide. In the case of YSZ as an electron-poor material this reduction step is necessarily hindered and might even control the complete kinetics.

For the case of $\mathrm{SrTiO}_{3}$ it has indeed been shown by Merkle et al. [7] that UV irradiation with photons of the same energy as the band gap leads to increased exchange rates of oxygen exposed samples. In a detailed analysis the authors prove that the electron transfer to the oxygen molecule is rate limiting at low and intermediate temperatures. In our case the band gap of YSZ equals $5.2 \mathrm{eV}$ [18], which corresponds to a wavelength of $238 \mathrm{~nm}$. The average kinetic energy of the electrons in the rf-plasma equals $10 \mathrm{eV}$, and thus, emission of UV photons with an energy of $5.2 \mathrm{eV}$ is at least theoretically possible. Nevertheless we can safely assume that electron-hole formation by UV irradiation plays a negligible role in the observed rate increase since the emission spectrum of our oxygen plasma shows no UV lines with such high energy. It has to be noted that even in the case of high energy UV emission the intensity of the emission would be much too low for a significant influence on the kinetics. As can be seen from Fig. 9, a rf-power of $25 \mathrm{~W}$ is already sufficient to see a serious rate increase. Merkle et al. had to use a $200 \mathrm{~W}$-discharge lamp in order to obtain an effect by UV irradiation.

\subsection{Particle bombardment/ion implantation}

In the stationary state of a plasma each surface of the plasma container and of the sample is charged negatively, and a significant potential difference between the plasma bulk and the wall is established. The plasma potential is positive referred to the wall potential. A positive space charge (double layer or Langmuir shield) is created, and equal fluxes of positive and negative particles towards the wall are maintained, where recombination of charged particles takes place. Only electrons with a high kinetic energy can cross the Langmuir shield, whereas the cations are accelerated by the electric field in the space charge region towards the wall. Lee et al. [26] estimate the average energy of bombarding ions as $6 T_{\mathrm{e}}$ and the energy of electrons as $2 T_{\mathrm{e}}$. In our case this corresponds to an average energy of $30 \mathrm{eV}$ of ions, which impinge the surface, and of $10 \mathrm{eV}$ of electrons. A cation energy of $30 \mathrm{eV}$ is in principle sufficient for the creation of defects at or close to the surface since it is higher than the binding energy in the crystal. But real ion implantation to the bulk requires energies higher than $0.5 \mathrm{keV}$ [34], thus can be excluded in our case. Ions with an energy of $30 \mathrm{eV}$ cannot penetrate the lattice deeper than $0.2 \mathrm{~nm}$, i.e. essentially not deeper than just one or two atomic layers. In essence, the cations from our rf-plasma hit the surface of any wall and might be incorporated into the first or second lattice plane but not into the crystal bulk.

Realising that ion implantation will definitely not participate in the process, it is nevertheless interesting to estimate the average flux of cations and electrons towards the surface under self-bias conditions, i.e. without applying an external voltage to the surface. According to Chapman [20] the plasma potential $V_{\mathrm{P}}$ is given as

$V_{\mathrm{P}}=\frac{T_{\mathrm{e}}}{2 e} \ln \left(\frac{m_{\mathrm{i}}}{2.3 m_{\mathrm{e}}}\right)+V_{\mathrm{f}}$

taking the Bohm criterion ${ }^{1}$ into account. Assuming an electron temperature $T_{\mathrm{e}}$ of $5 \mathrm{eV}$ and $\mathrm{O}^{+}$as the main ionic species, the plasma potential at a floating potential of $V_{\mathrm{f}}=0$ $\mathrm{V}$ equals $23.6 \mathrm{~V}$. This agrees perfectly with our measured plasma potentials, which are in the range of $20-25 \mathrm{~V}$.

A zirconia surface area of $1 \mathrm{~cm}^{2}$ includes approximately $8.8 \cdot 10^{14}$ atoms in the first atom layer. The ion flux $j_{\mathrm{i}}$ to the surface can be estimated by using the following equation:

$j_{i}=\frac{e n_{i} \overline{c_{i}}}{4}$

Assuming an ion temperature of $800 \mathrm{~K}$ and an ion density of $10^{10} / \mathrm{cm}^{3}$ (which corresponds to a degree of ionisation $\alpha=1.1 \cdot 10^{-6}$ ) we obtain a current density of 41.2

1 The ions entering the langmuir sheath have a directed velocity $u_{\mathrm{s}}$ which is greater than the minimum velocity $u_{\mathrm{B}}$. The velocity $u_{\mathrm{B}}$ is called Bohm velocity. 
$\mu \mathrm{A} / \mathrm{cm}^{2}$ and a corresponding flux of approximately $4 \times 10^{-10} \mathrm{~mol} /\left(\mathrm{cm}^{2} \mathrm{~s}\right)$. This flux can also be regarded as a rate of ion bombardment with 0.27 impacts per surface atom and second. In the exchange experiments the flux of ${ }^{18} \mathrm{O}$ across the surface approximates to $1 \times 10^{-8} \mathrm{~mol} /$ $\left(\mathrm{cm}^{2} \mathrm{~s}\right)$. Thus, the ${ }^{18} \mathrm{O}$ flux into the surface is definitely larger than the ionic flux to the surface and in consequence the ions can be excluded as possible reason for the faster surface kinetics.

\subsection{Surface heating/temperature effects}

In addition to the mere mass transfer effect of ion bombardment the simultaneous energy impact may lead to a local temperature increase and a corresponding acceleration of thermally activated surface reactions. Even more seriously, high energetic particles may transfer large amounts of energy to a small surface area close to the point of impact. These so-called hot spots have a rather short lifetime in the order of $10^{-10} \mathrm{~s}$ which is in the order of the period which is required for the hot spot to decay by lattice vibrations [35]. The influence of hot spots on the exchange cannot be estimated, thus we have to focus to the average energy transfer by all impinging particles. At least we can assume that the number of hot spots from high energetic ions will be small since the power of our rf-plasma is relatively low. On average every ion hitting the surface acquires an energy close to the product of its charge and the plasma potential. This results in an average energy flux of $12.5 \mathrm{~mJ} /\left(\mathrm{cm}^{2} \mathrm{~s}\right)$. The energy flux related to electron impact can be neglected.

The energy loss of the sample holder by heat radiation equals $270 \mathrm{~mJ} /\left(\mathrm{cm}^{2} \mathrm{~s}\right)$ at a temperature of $700 \mathrm{~K}$, thus we can safely assume that energy transfer by particle impact cannot change the sample temperature significantly. It is nontrivial to measure the temperature of the surface. For measurements with a conventional shielded thermocouple the heat capacity and conductivity of the thermocouple itself is too large. The temperature profile of the rf-reactor, which we measured with a shielded thermocouple, is comparable with measurements by Piejak et al. [36] in an Argon plasma generated in an ICP chamber. The temperatures increase with increasing pressure and rf-power. The smaller the diameter of the thermocouple, the higher is the temperature. A smaller thermocouple has a smaller metal shield, which results in a smaller thermal conductivity.

Measurements of the infrared emission are also excluded because the measured radiation is not only the radiation of the top surface layer. The lower layers emit, too. It depends on the transmission coefficient of the material, which average temperature is measured. We always measure a mean value. The best indicator is the diffusion coefficient received from the tracer experiments. As can be seen the diffusion coefficient is not changing significantly with increasing radio frequency power. Because the ion flux to the surface is very low and the lifetime of the hot spots generated by ion impact is very short, it is not expected that a higher surface temperature is the reason for the higher exchange rate.

A possible heating of the sample by the electromagnetic field can be excluded, too. The coupling of the electromagnetic field to the ionic material is relatively weak at the investigated temperatures. For example in the more powerful microwave heating an increase in the microwave absorption is only observed at temperatures of about $0.4-0.5$ $T_{\mathrm{m}}$ (where $T_{\mathrm{m}}$ is the melting temperature of the material) [37]. For zirconia the temperature should be definitely higher than $1000{ }^{\circ} \mathrm{C}$ in order to obtain serious heating effects.

\subsection{Sputter effects}

Highly energetic ions can initiate an impact cascade at the sample surface, leading finally to the emission of atoms from the surface. This effect is called sputtering. Sputtering of the sample surface by ion impact should lead to a continuous loss of material from the surface, i.e. of material with a high concentration of ${ }^{18} \mathrm{O}$. In effect, a lower concentration of ${ }^{18} \mathrm{O}$ and smaller exchange coefficients in the data evaluation should be the consequence.

Besides these arguments we do not expect any serious sputter effect in our experiments. YSZ is a hard material with a sputter yield being approximately 100 times lower than in the case of halides [38]. In addition the energy of impinging ions is relatively small. A check of the surface morphology after $24 \mathrm{~h}$ of plasma treatment did not show any measurable change with a laser interference microscope.

A related effect is based on the impact of electrons. If highly energetic electrons impinge at the surface ions can be desorbed. This process is called electron stimulated desorption (ESD). It usually requires electron energies in the order of a few $\mathrm{keV}$ [39]. As shown by the double probe measurements our electron energies are not higher than $10 \mathrm{eV}$, and thus, we neglect ESD as we also neglect sputtering by ion impact.

\subsection{Atomic oxygen}

Oxygen plasmas contain varying concentrations of oxygen atoms depending on the exact plasma properties. As Lee et al. [26] show it can even reach concentrations up to $50 \%$ depending on the plasma parameters. On the basis of our analytical methods we cannot determine the absolute concentration of oxygen atoms but assume that its concentration depends linearly on the plasma power as it does the degree of ionisation.

Oxygen atoms are unaffected by the existence of the space charge in front of the sample surface and can reach the sample surface without being slowed down or accelerated by the potential gradient in the space charge region. If we assume a concentration of oxygen atoms in the order of $1 \%$ 
their flux to the surface equals $1.2 \cdot 10^{-5} \mathrm{~mol} /\left(\mathrm{cm}^{2} \mathrm{~s}\right)$ under typical plasma conditions. This value is considerably higher than the flux of oxygen ions and electrons, and thus, is probably more important than any rate increase caused by the impact of ionic species.

It has been shown by Manning et al. [13] that the ratedetermining step of oxygen exchange above $700{ }^{\circ} \mathrm{C}$ is the dissociation of the oxygen molecule. We assume that at lower temperatures the incorporation and reduction step may take over the role of the rate-limiting process.

\subsection{Surface charging/electric field effect}

The formation of negative surface charges at plasma walls is one the most prominent plasma effects. As we estimated above, the flux of electrons to the YSZ surface can be estimated as approximately $4 \cdot 10^{-10} \mathrm{~mol} /\left(\mathrm{cm}^{2} \mathrm{~s}\right)$, and the surface charge is typically in the order of $1.7 \times 10^{-11}$ $\mathrm{C} / \mathrm{cm}^{2}$ at a plasma potential of $20 \mathrm{~V}$, thus representing a relatively low surface charge density.

The influence of the negative surface charge can be three-fold:

(a) The electrons, which create the negative surface charge, may occupy surface states in or close to the conduction band, which then promote the reduction of adsorbed oxygen atoms. It is interesting to note that this effect is probably comparable to the formation of electron and holes at oxide surfaces by UV irradiation. In any case a rate increase is related to a lift in the electron concentration at the surface, which directly influences the reduction of oxygen species. Therefore we refer to this effect as a concentration effect. It is not possible to prove this effect or to make a quantitative statement on its influence. Whether a negative surface charge has also an effect on the oxidation of oxygen ions, which are leaving the crystal during the exchange experiment, is difficult to decide. Currently we assume that any effect on the oxygen release can be neglected.

(b) Additionally to point (a) the negative surface charge establishes a positive space charge under the oxide surface. Only mobile positive oxygen vacancies can form this space charge leaving (relatively) negatively charged dopand cations behind. The Debye length of YSZ is very short due to the high degree of doping. Thus the concentration of anion vacancies should only be increased within a distance of approximately $0.1 \mathrm{~nm}$ close to the surface. We believe that this increased vacancy concentration may also participate in an increased oxygen incorporation which requires vacancies at the surface, but cannot give a quantitative measure. Regarding the estimate of the surface charge, the increase of the vacancy concentration is negligible compared to the mean vacancy concentration.

Kilner et al. [40] have shown that the exchange coefficient $k$ can be correlated to the diffusion coefficient D.

$\log k=m \cdot \log D+b$
For mixed conductors the coefficient $m$ is approximately 0.5 , for oxides with the fluorite structure the coefficient is approximately 1 [41]. The diffusion coefficient $D$ itself is proportional to the vacancy concentration which depends on the material properties and the dopand concentration. $D_{\mathrm{v}}$ is the vacancy diffusion coefficient.

$D=\left[\mathrm{V}_{\ddot{\mathrm{o}}}\right] \cdot D_{\mathrm{v}}$

This means that the rate constant for the oxygen exchange is related to the oxygen vacancy concentration if the surface charge creates a significant vacancy space charge. This might explain higher $k$ values for the exchange in materials with more pronounced space charge effects.

As the concentration effect for electrons in (a) the space charge effect can also be regarded as a (small) concentration effect on the surface exchange rate.

(c) In addition to the effect of a negative surface charge on the concentrations of electrons and oxygen vacancies as reaction partners in the rate-limiting surface reaction, a real electric field effect might also assist in the exchange kinetics. As is well known from electrode kinetics in the liquid state, an electric field across an electrode interface will increase the rate of either the cathodic or anodic transfer process. In our case the oxide surface possesses two asymmetric space charges in the solid electrolyte and the plasma phase. The electric field vector in the solid electrolyte will point from the bulk to the surface, and it might be that thermally activated jumps or transfer steps of negative species (oxygen ions) are supported. Again, we cannot give a quantitative estimation of this effect in the current state of investigation.

Perhaps it is worthwhile to investigate the interaction between a solid electrolyte and a plasma more thoroughly by theoretical methods.

Summarizing all above discussed effects, we believe that the negative surface charge of the YSZ surface in the rfplasma contributes to the observed rate increase. We cannot exclude a contribution of oxygen atoms but have no means to give a quantitative answer.

\section{Conclusions}

Isotopic oxygen exchange experiments were performed in rf-oxygen plasma. In contrast to the exchange with molecular oxygen the exchange rate is up to 100 times higher for samples immersed in an oxygen plasma, even at low plasma powers. In the temperature range $500-600{ }^{\circ} \mathrm{C}$ the exchange rate is not increasing significantly with the exchange temperature.

Several effects related to the plasma/solid interaction are discussed as possible causes for the rate increase. Currently we assume that the strong negative charging of a sample surface plays a dominant role for the rate 
increase. The negative surface charge is a consequence of electronic surface states, which may promote the slow reduction of oxygen species, and also leads to a space charge in the electrolyte being composed of anion vacancies. It might be that this space charge with an increased concentration of vacancies also promotes the oxygen incorporation. An influence of oxygen atoms on the rate increase can neither be proved nor excluded at the present state.

\section{Acknowledgements}

This work was supported by the german research foundation with a Gerhard Hess project (projects DFG Ja 648/4-1 and DFG Ja 648/4-2).

\section{References}

[1] B.C.H. Steele, Solid State Ionics 86-88 (1996) 1223-1234

[2] J.A. Lane, S.J. Benson, D. Waller, J. Kilner, Solid State Ionics 121 (1999) 201-208.

[3] C.-R. Song, H.-I. Yoo, Solid State Ionics 120 (1999) 141-153.

[4] A. Mitterdorfer, L.J. Gauckler, Solid State Ionics 117 (1999) 187-217.

[5] R.J. Chater, S. Carter, J.A. Kilner, B.C.H. Steele, Solid State Ionics 53-56 (1992) 859-867.

[6] M.W. den Otter, B.A. Boukamp, H.J.M. Bouwmeester, Solid State Ionics 139 (2001) 89-94.

[7] R. Merkle, R.A. De Souza, J. Maier, Angew. Chem. 113 (2001) 2184-2187.

[8] J. Maier, Solid State Ionics 112 (1998) 197-228.

[9] C. Li, K. Domen, K. Maruya, T. Onishi, J. Am. Chem. Soc. 111 (1989) $7683-7687$.

[10] B.A. Boukamp, H.J.M. Bouwmeester, H. Verweij, A.J. Burggraaf, Mater. Res. Soc. Symp. Proc. 293 (1993) 361-366.

[11] T. Bak, M. Rekas, J. Nowotny, C.C. Sorell, A. Adamski, K. Dyrek, Z. Sojka, Ionics 7 (2001) 332-338.

[12] B. Luerssen, J. Janek, S. Guenther, M. Kiskinova, R. Imbihl, Phys. Chem. Chem. Phys. 4 (2002) 2673-2679.

[13] P.S. Manning, J.D. Sirman, J.A. Kilner, Solid State Ionics 93 (1997) $125-132$.

[14] P.S. Manning, J.D. Sirman, R.A. De Souza, J.A. Kilner, Solid State Ionics 100 (1997) 1-10.
[15] J.T. Gudmundsson, M.A. Lieberman, Plasma Sources Sci. Technol. 7 (1998) $1-12$.

[16] M. de Ridder, A.G.J. Vervoort, R.G. van Welzenis, H.H. Brongersma, Solid State Ionics 156 (2003) 255-262.

[17] J.H. Park, R.N. Blumenthal, J. Electrochem. Soc. 136 (1989) 2867.

[18] U. Vohrer, H.-D. Wiemhöfer, W. Göpel, Solid State Ionics 59 (1993) $141-149$.

[19] M. de Ridder, R.G. van Welzenis, H.H. Brongersma, Surf. Interface Anal. 33 (2002) 309-317.

[20] B. Chapman, Glow Discharge Processes, Wiley, New York, 1980.

[21] J.D. Swift, M.J.R. Schwar, Electrical Probes for Plasma Diagnostics, Ilife Books, London, 1970.

[22] E.O. Johnson, L. Malter, Phys. Rev. 80 (1950) 58-68.

[23] S. Pfau, M. Tichy, Low Temperature Plasma Physics, Wiley-VCH, Berlin, 2000

[24] F.F. Chen, Plasma Diagnostic Techniques, Academic Press, New York, 1965, pp. 177-183

[25] G. Janzen, Plasmatechnik, Hüthig Verlag, Heidelberg, 1992.

[26] C. Lee, D.B. Graves, M.A. Lieberman, D.W. Hess, J. Electrochem. Soc. 141 (1994) 1546-1555.

[27] E.J.H. Collart, J.A.G. Baggerman, R.J. Visser, J. Appl. Phys. 70 (1991) 5278-5281.

[28] R.E. Walkup, K.L. Saenger, G.S. Selwyn, J. Chem. Phys. 84 (1986) 2668-2674.

[29] J. Crank, The Mathematics of Diffusion, Oxford Sci. Publ., Oxford, 1975.

[30] I. Denk, F. Noll, J. Maier, J. Am. Ceram. Soc. 80 (1997) 279.

[31] M.A. Lieberman, A.J. Lichtenberg, Principles of Plasma Discharges and Materials Processing, Wiley, New York, 1994.

[32] A.E. Hughes, Am. Ceram. Soc. 78 (1995) 369-378.

[33] M. de Ridder, The Rate-Limiting Factor of the Solid Oxide Fuel Cell, $\mathrm{PhD}$ thesis, Technical University of Eindhoven, Eindhoven (2002), Chap. 3.

[34] A. Anders, Handbook of Plasma Immersion Ion Implantation and Deposition, Wiley, New York, 2000.

[35] R. Kelly, Ion Bombardment Modification of Surfaces, Elsevier, Amsterdam, 1984.

[36] R. Piejak, V. Godyak, B. Alexandrovich, N. Tishchenko, Plasma Sources Sci. Technol. 7 (1998) 590-598.

[37] Y.V. Bykov, K.I. Rybakov, V.E. Semenov, J. Phys. D: Appl. Phys. 34 (2001) R55-R75.

[38] R. Kelly, Surf. Sci. 90 (1979) 280-318.

[39] J.A. Kilner, L. Ilkov, Vacuum 34 (1984) 139-143.

[40] J.A. Kilner, R.A. De Souza, I.C. Fullarton, Solid State Ionics 86-88 (1996) 703-709.

[41] J.A. Kilner, in: T.A. Ramanarayana, W.C. Worrell (Eds.), Proc. of the 2nd Intern. Symp. on Ionic and Mixed Conducting Ceramics, Electrochem. Soc. Proc. vol. 94-12, (1994) 174-190. 